\title{
Quantitative trait loci linked to thalamus and cortex gray matter volumes in BXD recombinant inbred mice
}

\author{
H Dong ${ }^{1}$, MV Martin ${ }^{1}$, J Colvin ${ }^{1}$, Z Ali ${ }^{1}$, L Wang ${ }^{1}$, L Lu ${ }^{2,3}$, RW Williams ${ }^{3}$, GD Rosen ${ }^{4}$, JG Csernansky ${ }^{1,5}$ \\ and JM Cheverud ${ }^{5}$ \\ ${ }^{1}$ Department of Psychiatry, Washington University School of Medicine, St Louis, MO, USA; ${ }^{2}$ Key Laboratory of Nerve Regeneration, \\ Nantong University, Nantong, China; ${ }^{3}$ Department of Anatomy and Neurobiology, University of Tennessee Health Science Center, \\ Memphis, TN, USA; ${ }^{4}$ Department of Neurology, Beth Israel Deaconess Medical Center, Boston, MA, USA and ${ }^{5}$ Departments of \\ Anatomy and Neurobiology, Washington University School of Medicine, St Louis, MO, USA
}

To investigate whether there are separate or shared genetic influences on the development of the thalamus and cerebral cortex, we identified quantitative trait loci (QTLs) for relevant structural volumes in BXD recombinant inbred (RI) strains of mice. In $34 \mathrm{BXD} R \mathrm{R}$ strains and two parental strains (C57BL/ $6 \mathrm{~J}$ and $\mathrm{DBA} / 2 \mathrm{~J})$, we measured the volumes of the entire thalamus and cortex gray matter using point counting and Cavalieri's rule. Heritability was calculated using analysis of variance (ANOVA), and QTL analysis was carried out using WebQTL (http://www.genenetwork.org). The heritability of thalamus volume was $36 \%$, and three suggestive QTLs for thalamus volume were identified on chromosomes 10, 11 and 16 . The heritability of cortical gray matter was $43 \%$, and four suggestive QTLs for cortex gray matter volume were identified on chromosomes 2, 8, 16 and 19. The genetic correlation between thalamus and cortex gray matter volumes was 0.64 . Also, a single QTL on chromosome 16 (D16Mit100) was identified for thalamus volume, cortex gray matter volume and Morris water maze search-time preference $(r=0.71)$. These results suggest that there are separate and shared genetic influences on the development of the thalamus and cerebral cortex.

Heredity (2007) 99, 62-69; doi:10.1038/sj.hdy.6800965; published online 4 April 2007

Keywords: thalamus volume; cortex gray matter volume; quantitative trait locus; BXD recombinant inbred mice; schizophrenia

\section{Introduction}

There are numerous structural and functional connections between the thalamus and cortex in the mammalian brain (Krettek and Price, 1977; Carvell and Simons, 1987; Groenwegen, 1988; Johnson and Alloway, 1994; Scannell et al., 1999; Shipp, 2003; Matsuzaki et al., 2004). Of particular interest to scientists studying the neurobiology of neuropsychiatric disorders are the dense reciprocal connections between the ventral anterior and mediodorsal nuclei of the thalamus and the prefrontal cortex, which support executive cognition functions, such as working memory, selective attention and planning (Kievit and Kuypers, 1977; Jones and Friedman, 1982; Ilinsky and Kultas-Ilinsky, 1987; Jones, 1997). Moreover, deficits in fronto-striato-thalamic and fronto-thalamiccerebellar circuits have been implicated in mediating the cognitive deficits of neuropsychiatric disorders, such as schizophrenia (Volk and Lewis, 2003; James et al., 2004; Mendrek et al., 2004).

Correspondence: Dr H Dong, Department of Psychiatry, Washington University School of Medicine, Campus Box 8134, 660 S Euclid Avenue, St Louis, MO 63110, USA.

E-mail: dongh@wustl.edu

Received 2 May 2006; revised 27 February 2007; accepted 28

February 2007; published online 4 April 2007
Recent studies of the normative variability of brain structure suggest that variations in cerebral cortex volume and shape are under substantial genetic control (Thompson et al., 2001a, b; Winterer and Goldman, 2003). Using detailed three-dimensional maps, (Thompson et al., 2001a, b) have shown that genetic factors significantly influence cortical folding in Broca's and Wernicke's language areas, as well as in frontal brain regions (Thompson et al., 2001a,b). However, whether genetic factors that influence cortical development also have an impact on the development of related thalamus structures is far less clear.

One approach to dissecting the genetic basis of complex structural and behavioral phenomena has been to identify associations between specific chromosomal locations and specific phenotypic traits. Chromosomal locations with quantitative effects on phenotypic traits are referred to as quantitative trait loci (QTLs) (Doerge, 2002; Chesler et al., 2004, 2005). While the ultimate goal of such studies is to identify individual genes that influence a specific phenotypic trait, QTL mapping can limit the search for candidate genes to a restricted region of the genome (Korstanje and Paigen, 2002). In the case of the mouse genome, this region would ideally be on the order of $0.5-1.5 \mathrm{cM}$ (about 10-20 genes). GeneNetwork (Wang et al., 2003a) is a program developed for sophisticated QTL mapping and enables rapid gene analysis at many levels - from gene expression to genetic networks for 
brain and behavior. In this study, we used GeneNetwork to search for separate and shared QTLs that influence the volumes of thalamus and cortex gray matter. Further, we examined structure - function relationships related to these QTLs using the archival behavioral data available in the GeneNetwork database.

\section{Materials and methods}

\section{Subjects}

One hundred and eighty $B X D$ recombinant inbred mice and C57BL/6J and DBA/2J parental animals (95 males and 85 females, totaling 36 strains) were used in this study $(N=5.0$ animals per strain; age range from 31 to 493 days; see Figure 1 for strain means and standard errors). Digital images of mouse brain sections were obtained from the Mouse Brain Library (MBL, http:// www.mbl.org). MBL procedures for processing tissue have been described previously (http://www.mbl.org) (Zygourakis and Rosen, 2003). Briefly, selected animals were deeply anesthetized with Avertin $(0.5-0.8 \mathrm{ml}$, intraperitoneally (i.p.)) and transcardially perfused with $0.9 \%$ phosphate-buffered saline followed by approximately $15 \mathrm{ml}$ of $1.25 \%$ glutaraldehyde and $1.0 \%$ paraformaldehyde in $0.1 \mathrm{M}$ phosphate buffer. An additional $10-20 \mathrm{ml}$ of double-strength fixative (2.5\% glutaraldehyde and $2.0 \%$ paraformaldehyde in the same buffer) was subsequently perfused. The head was removed and put in the final fixative until the brain was dissected. Individual brains were embedded in $12 \%$ celloidin and sectioned in the coronal plane at $30 \mu \mathrm{m}$. Ten sets of coronal serial sections were cut per brain. From these 10 sets, four sets (the first, second, sixth and seventh series) were selected for cresyl violet staining. The rest of the sets were kept for later use. For this study, we chose the first and second of the four sets stained with cresyl violet to measure the entire thalamus volume (average 7-11 sections/animal in each set; the interval between measured sections was $0.3 \mathrm{~mm}$ ) and cortex gray matter volume (average 11-14 sections/animal in each set; the interval between measured sections was $0.6 \mathrm{~mm}$ ). For data analysis, the volumes obtained by assessing the two sets for each animal were averaged, except in rare cases where data from only one set were available.

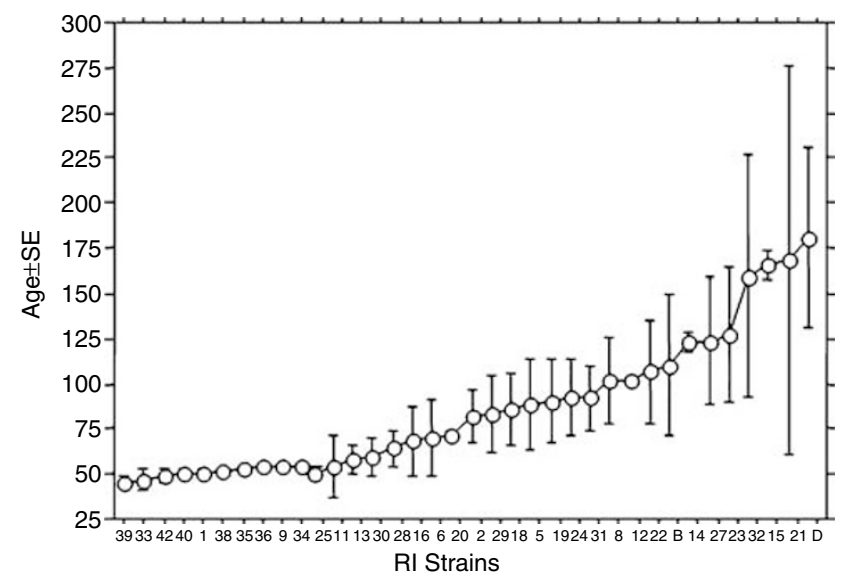

Figure 1 Interaction line plots showing variation (means and standard errors) in age across all RI strains.

\section{Volume measurement}

Digital images of cresyl violet-stained coronal sections of mouse brains were downloaded from the MBL in JPEG format. The final resolution of the images was one pixel $^{2}=0.000685 \mathrm{~mm}^{2}$, and the images were imported into Analyze 7.0 for assessment. Using the Analyze 7.0 stereology module, a grid was randomly placed over each section. The area of the thalamus and cortex gray matter was measured in each coronal section using Cavalieri's principle. The contours of the thalamus and cortex gray matter were identified using landmarks derived from a mouse brain atlas (Paxinos and Franklin, 2001). The points on the grid were spaced 8 pixels apart on the $X$ and $Y$ axes. Thus, the total number of grid points over the target area was multiplied by 64 to obtain the total pixels encompassing the thalamus or cortex gray matter in each brain. This number was then multiplied by $0.000685 \mathrm{~mm}^{2}$ and $0.3 \mathrm{~mm}$ (for thalamus) or $0.6 \mathrm{~mm}$ (for cortex) to obtain the volumes $\left(\mathrm{mm}^{3}\right)$.

The shrinkage among the cases in MBL is variable but can be estimated (Williams, 2000). To correct for variance due to shrinkage caused by histological processing, we divided thalamus and cortex gray matter volumes by total brain volume and then multiplied the result by the brain volume expected for a known brain weight, as described by Peirce et al. (2003). Brain volumes and weights for each animal were obtained from the MBL database (Williams, 2000).

Backward elimination linear regression was used to determine whether age or sex predicted thalamus and cortex gray matter volumes. Neither age nor sex significantly predicted thalamus volume. Age $(\mathrm{F}=4.98$, $P<0.05)$, but not sex, significantly predicted cortex gray matter volume. Age was negatively correlated with cortex gray matter volume $(r=-0.17)$. Therefore, linear regression was used to adjust for age-related variation in cortex gray matter volume.

We also examined structure-specific genetic influences on thalamus and cortex gray matter volumes. Because the thalamus and cortex each account for a large proportion of whole brain volume in the rodent, we did not remove variance associated with whole brain volume, as has been done previously (Peirce et al., 2003). Instead, to assess structure-specific influences, we performed backward linear regression analyses for both thalamus and cortex gray volumes, using the variable 'brain volume minus structure of interest volume (thalamus or cortex gray matter)' as a covariate.

\section{QTL mapping}

Marker regression analyses were performed using the GeneNetwork. One-way ANOVAs were run using 781 microsatellite markers and BXD strain means for the volumes of interest. To assess genome-wide significance levels corrected for multiple comparisons, each peak likelihood ratio statistic (LRS) was compared to the 5\% threshold LRS computed for 2000 permutations. The four sets of analyses were based on strain means for the four volume variables: (1) thalamus volume strain means; (2) thalamus volume corrected for 'total brain volume minus the thalamus volume'; (3) cortex gray matter agecorrected strain means; and (4) cortex gray matter volume corrected for 'total brain volume minus cortex gray matter volume'. For each of the four variables, one 
regression analysis was run. For thalamus volume, the suggestive $\mathrm{LRS}=9.49$ and the significant $\mathrm{LRS}=15.60$, based on randomization. For cortex gray matter, the suggestive LRS $=9.56$ and the significant LRS $=16.09$. As an exploratory effort, we correlated BXD behavioral phenotypes, which have been implicated in the functions of thalamus and cortex, with each of the four neuroanatomical traits using GeneNetwork.

\section{Results}

Volumes in the parental strains, corrected for shrinkage, were as follows: thalamus - C57BL/6J $\left(19.194 \pm 0.774 \mathrm{~mm}^{3}\right), \mathrm{DBA} / 2 \mathrm{~J}\left(16.097 \pm 0.450 \mathrm{~mm}^{3}\right)$; cortex gray matter - C57BL/6J $(128.39 \pm 22.19)$, DBA/2J $(87.83 \pm 10.16)$. Both of these traits were significantly different in the parental strains (thalamus: $F=11.06$, $\mathrm{df}=1,13, P=0.005$; cortex gray matter $\mathrm{F}=22.05, \mathrm{df}=1$, $13, P=0.0004)$. Among the BXD RI strains, there was a significant strain effect on thalamus volume (ANOVA, $\mathrm{F}=3.89, \mathrm{df}=33,131 ; P<0.001)$ and cortex gray matter volume (ANOVA, $\mathrm{F}=2.88, \mathrm{df}=33,128 ; P<0.001)^{\prime}$. Figure 2 summarizes thalamus and cortex gray matter volumes for all RI strains (means and standard deviations). There was a significant correlation between these two volume measures across strain means (Pearson's $r=0.55 ; P=0.0007)$. There was no effect of strain on the estimated amount of tissue shrinkage (ANOVA $F=1.27$; $\mathrm{df}=35,144 ; P>0.05)$.

\section{Heritability of thalamus and cortex gray matter volume measurements}

Broad-sense heritability was calculated using age-corrected (when appropriate) and shrinkage-corrected volumes. Heritability was calculated using an $R^{2}$ statistic (Falconer and Mackay, 1997) as follows:

$$
\begin{aligned}
H^{2} & =\frac{M S_{\text {strain }}-M S_{\text {error }}}{M S_{\text {strain }}+\left(n_{0}-1\right) M S_{\text {error }}} \\
n_{0} & =\frac{1}{N-1}\left[\sum_{i=1}^{N} n_{i}-\left(\sum_{i=1}^{N} n_{i}\right) \sum_{i=1}^{N} n_{i}\right]
\end{aligned}
$$

$N$ is the number of strains and $n_{i}$ is the number of individuals in the $i$ th strain.

In BXD mice, heritabilities of thalamus volume and cortex gray matter volume were $36 \%$ (s.e. $=0.084$ ) and $43 \%$ (s.e. $=0.085)$, respectively.

\section{Genetic correlation between thalamus and cortex gray matter volumes}

The genetic correlation between thalamus and cortex gray matter volumes was calculated as follows:

$$
R_{G}=\operatorname{cov}(C G M, T h) \sqrt{ }(V(C G M) * V(T h))
$$

where $T h$ indicates thalamus and CGM indicates cortex gray matter (Falconer and Mackay 1997). The genetic correlation between thalamus and cortex gray matter volumes was 0.64 (s.e. $=0.075$ ), indicating the likelihood of pleiotropic effects of genes on these two traits.

\section{QTL mapping}

Suggestive QTLs were defined as those with a $P$-value of less than 0.05 , based on randomization results. Significant QTLs were defined as those with a randomizationbased $P$-value of less than 0.01. Three QTLs were
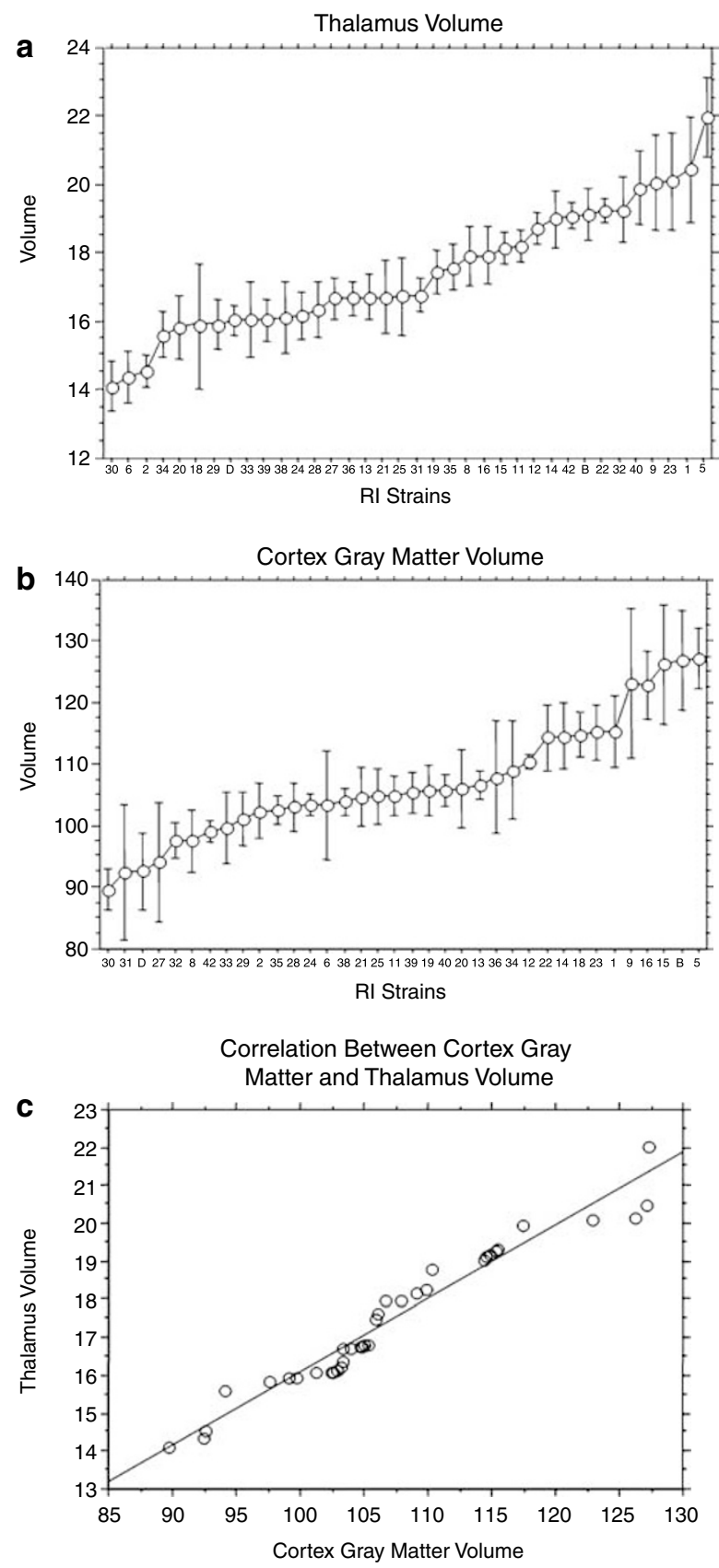

Figure 2 Interaction line plots summarizing the variability of structural volumes across RI strains (means and standard deviation) (see (a) for thalamus volumes and (b) for cortex gray matter volumes). Strains are ordered by feature volume. Panel c represents the correlation between the two volume measures across the strain means.

identified for thalamus volume on chromosomes 10, 11 and 16 (see Figure 3; Table 1). After removing variance associated with 'total brain volume minus thalamus volume', all QTLs remained. Four QTLs for cortex gray matter volume were located on chromosomes 2, 8, 16 and 19 (see Figure 3; Table 1). After adjusting cortex gray matter volume for 'total brain volume minus cortex gray matter volume', all QTLs remained.

We examined the correlations between QTL markers for each variable to detect genomic locations spuriously correlated by non-syntenic association (Williams et al., 

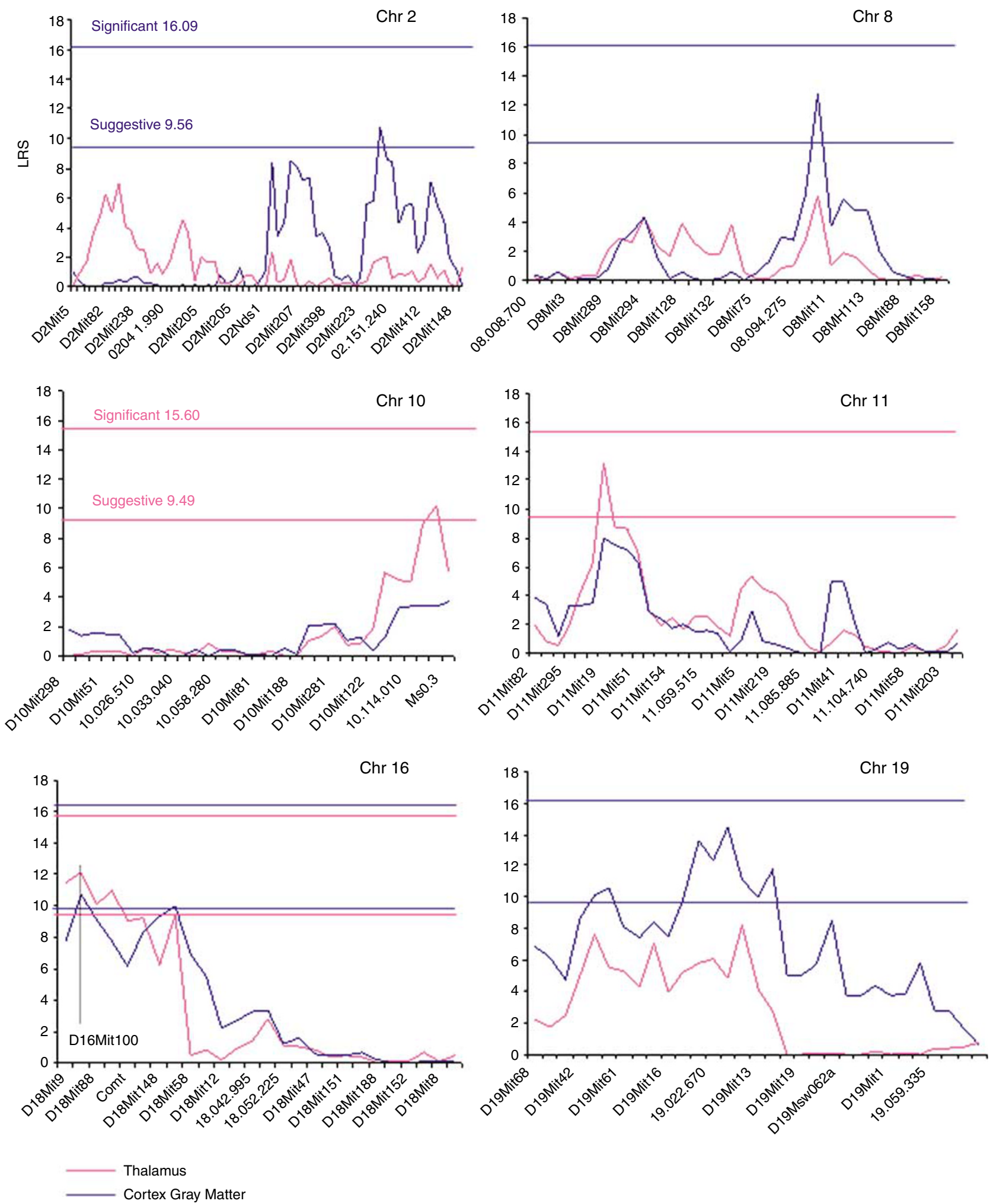

Figure 3 LRS scores for structural measures. Pink lines illustrate the LRS scores for uncorrected thalamus volumes. Three QTLs were identified on chromosomes 10, 11 and 16. Blue lines illustrate the LRS scores for cortex gray matter volume strain means adjusted for age. Four QTLs were identified on chromosomes 2, 8, 16 and 19. The $Y$ axis represents the LRS scores as determined by marker regression at each microsatellite marker along the $X$ axis. LRS score $=1 / 4.6$ the logarithm of the odds ratio (the LOD score). The data are presented as LRS plots with lines connecting the intervals between the LRS data points. A single QTL (peak at D16Mit100, indicated by the dotted black line in panel 5) on chromosome 16, was associated with both thalamus and cortex gray matter volumes. 
2001; Cheverud et al., 2004). Non-syntenic association may occur when unlinked regions of the genome are associated with one another by chance in a limited sample of RI strains. This can result in a single true QTL having apparent effects at two different genomic locations. These 'shadow' QTLs may arise either as a result of random fixation of alleles on different chromosomes during the production of RI strains or as a result of selection for particular combinations of alleles on different chromosomes. Non-syntenic intermarker correlations beyond $r= \pm 0.51$ were considered as large enough to result in 'shadow' QTLs among non-syntenic locations. This threshold was obtained by multiplying the expected standard deviation of the correlation frequency distribution around the mean of zero, or the square root of $(1 /($ strain number -1$))$, by three. Thus, correlations more extreme than 0.51 are more than three standard deviations from the mean expected under independent segregation. In this study, there were no significant correlations between markers identifying unlinked QTLs.

\section{Correlation between thalamus and cortex gray matter} volumes and behavioral phenotypes

We correlated archival behavioral data available on the GeneNetwork with measures of thalamus and cortex gray matter volumes in the BXD strains. The selected behaviors included spatial memory (Morris water maze performance), fear conditioning, exploration during the light-dark paradigm, exploratory locomotor activity and sensorimotor gating. Our results indicated a strong correlation between thalamus volume and spatial memory (i.e., search time preference in the Morris water maze paradigm $n=11 ; r=-0.84, P<0.001$; Wehner et al., 1990). Moreover, a single QTL (peak at D16Mit100) was associated with thalamus volume, cortex gray matter volume (see Figure 3, panel 5) and Morris water maze search-time preference $(r=0.71, P<0.05)$.

\section{Correlation between thalamus and cortex gray matter volumes and brain gene expression}

Using gene expression data available at WebQTL, we correlated thalamus and cortex gray matter volumes with the pattern of brain-related gene expression across the BXD strains to determine whether any brain-related gene expression QTLs mapped to the same locations as our morphological QTLs. The results are shown in Tables 2 and 3 for the thalamus and cortex gray matter, respectively. Cortex gray matter volume was highly correlated $(r=0.59, P=0.0024)$ with amurine thymoma viral oncogene homologue (Akt1), a protein kinase that has been shown to have an important role in neurodevelopment (Wang et al., 2003b) and working memory formation (Mizuno et al., 2003). Also, the Akt1 gene is located within the interval of the QTL we identified on chromosome 19, and its expression was also significantly

Table 1 Linkage statistic for thalamus and cortex gray matter volume phenotypes

\begin{tabular}{|c|c|c|c|c|c|c|c|c|c|c|c|}
\hline Trait & $Q T L$ & Proximal & Distal & Chr & Effect size & Effect size/s.d. & LRS & $L O D$ & Proximal $(\mathrm{Mb})$ & Distal $(M b)$ & P-value \\
\hline \multirow[t]{3}{*}{ Thalamus volume } & D10Mit14 & 10.114 .010 & Ms6-3 & 10 & 1.932 & 0.870 & 10.217 & 2.22 & 114.863 & 119.356 & 0.0060 \\
\hline & D11Mit19 & 11.020 .100 & D11Mit51 & 11 & 2.110 & 0.951 & 13.177 & 2.86 & 20.849 & 36 & 0.0013 \\
\hline & D16mit100 & D16Mit9 & D16Mit146 & 16 & 2.006 & 0.903 & 12.101 & 2.63 & 6.329 & 23.570 & 0.0023 \\
\hline \multirow{4}{*}{$\begin{array}{l}\text { Cortex gray matter } \\
\text { volume }\end{array}$} & D2Mit42 & D2Mit340 & D2Mit493 & 2 & 12.378 & 1.097 & 10.68 & 2.32 & 148.315 & 153.430 & 0.0048 \\
\hline & D8Mit312 & 08.094 .275 & D8Mit11 & 8 & 12.842 & 1.1 & 12.87 & 2.80 & 92.292 & 77 & 0.0016 \\
\hline & D16Mit100 & D16Mit9 & D16Mit167 & 16 & 12.186 & 1.080 & 10.73 & 2.33 & 6.329 & 33.983 & 0.0046 \\
\hline & D19Mit40 & D19Mit16 & D19Mit63 & 19 & 13.448 & 1.192 & 14.41 & 3.13 & 19.593 & 35.382 & 0.0007 \\
\hline
\end{tabular}

QTLs that associated with anatomical traits; proximal and distal-markers within a one LOD score drop on the proximal and distal portion of QTL; Chr, the chromosome containing QTL; effect, an estimate of the magnitude of gene. Effect size, the effect size is derived by multiplying the additive effect size by 2 . Effect size/s.d., the effect size relative to the amount of variation of each trait; LRS, the likelihood ratio statistic; LOD, logarithm of the odds ratio.

Table 2 QTLs for thalamus volume and brain gene expression

\begin{tabular}{|c|c|c|c|c|}
\hline QTLs & Related gene & Phenotype & Correlation & P-value \\
\hline \multirow[t]{2}{*}{ Chr10 (D10Mit14) } & \multirow[t]{2}{*}{ 2310043D08Rik } & Brain weight & 0.6405 & 0.004 \\
\hline & & Total hippocampus volume & 0.5063 & 0.015 \\
\hline \multirow{7}{*}{ Chr11 (D11Mit19) } & \multirow[t]{3}{*}{ vamp2 } & Forebrain weight & 0.5430 & 0.0053 \\
\hline & & Striatum volume & 0.4419 & 0.0296 \\
\hline & & Morris water maze $\log$ latency 2 & -0.4918 & 0.0439 \\
\hline & \multirow[t]{4}{*}{ 4931428D14Rik } & Brain weight & 0.5840 & 0.0123 \\
\hline & & Stratum volume & 0.4419 & 0.0296 \\
\hline & & Dopamine transporter expression in frontal cortex & 0.6389 & 0.0324 \\
\hline & & Morris water maze log latency 2 & -0.4918 & 0.0439 \\
\hline \multirow{5}{*}{ Chr16 (D16Mit100) } & \multirow{5}{*}{ Fgd4 } & Volume of amygdala & -0.6904 & $6.85 \mathrm{e}-05$ \\
\hline & & Hippocampus volume & -0.6858 & 0.00025 \\
\hline & & Forebrain weight & -0.5493 & 0.00466 \\
\hline & & Morris water maze site preference & 0.8864 & 0.00496 \\
\hline & & Prepulse inhibition of the acoustic startle response & -0.6521 & 0.00498 \\
\hline
\end{tabular}

Selected genes located within thalamus volume QTLs. These genes were selected because their expression was significantly correlated with thalamus volume. Further, WebQTL analyses revealed that gene expression of the selected genes was also correlated with other measures of brain structure and/or behavioral performance. 
Table 3 QTLs for cortical gray matter volume correlated with brain gene expression

\begin{tabular}{|c|c|c|c|c|}
\hline QTLs & Related gene & Phenotype & Correlation & P-value \\
\hline \multirow[t]{3}{*}{ Chr2 (D2Mit42) } & 2900060K15Rik & Forebrain weight & -0.6044 & 0.047 \\
\hline & & Prepulse in prepulse inhibition of the acoustic startle response & 0.5001 & \\
\hline & Flrt3 & Brain weight & -0.5688 & 0.0097 \\
\hline \multirow{4}{*}{ Chr8 (D8Mit312) } & 4432417N03Rik & Brain weight & -0.7999 & 3.96-05 \\
\hline & & Hippocampus volume & -0.5508 & 0.00692 \\
\hline & & Morris water maze & 0.8654 & 0.00856 \\
\hline & & Search-time preference cerebellum fissure frequency & 0.5139 & 0.03357 \\
\hline \multirow{2}{*}{ Chr16 (D16Mit100) } & Tnfrsf17 & Dopamine transporter expression in frontal cortex & -0.7609 & 0.00479 \\
\hline & & Prepulse inhibition of the acoustic startle response & -0.5239 & 0.02426 \\
\hline \multirow[t]{3}{*}{ Chr19 (D19Mit40) } & C130032J12Rik & Brain weight & -0.5656 & 0.00520 \\
\hline & & Morris water maze site preference & 0.8667 & 0.00830 \\
\hline & & Striatum volume & -0.4834 & 0.01567 \\
\hline
\end{tabular}

Selected genes located within cortex gray matter volume QTLs. These genes were selected because their expression was significantly correlated with cortex gray matter volumes. Further, WebQTL analyses revealed that gene expression of the selected genes was also correlated with other measures of brain structure and/or behavioral performance.

associated with genotype at the peak marker D19Mit40 of this QTL $(\mathrm{LRS}=10.19$; effect size $=0.49)$.

\section{Discussion}

The work we present here is the first to compare the pattern of genetic influences on thalamus and cortex gray matter volumes in recombinant inbred strains mice. We hypothesized that there may be shared genetic influences on the development of these structures, and therefore on their volumes, because of the numerous structural and functional relationships between them. We found three QTLs for thalamus volume on chromosomes 10, 11 and 16 , and four QTLs for cortex gray matter volume on chromosomes 2, 8, 16 and 19. A cortex gray matter volume QTL and a thalamus volume QTL on chromosome 16 shared a common peak marker (D16Mit100). When variation associated with 'total brain volume minus structure of interest' was removed, all QTLs remained, indicating that these QTLs were not simply due to the effects of these QTLs on overall brain volume. Although this does not rule out the possibility that the volume of other brain structures may be influenced by one or more of these QTLs, the data suggest that there are both shared and separate genetic influences on thalamus and cortex gray matter volumes in BXD RI mice.

We found an age-related decrease in cortex gray matter volume, which is consistent with the literature related to human(Walhovd et al., 2005). There was no age effect on thalamus volume. Reports of age effects on thalamus volume in human have been mixed, with studies finding age-related decreases or no change in thalamus volume (Sullivan et al., 2004; Walhovd et al., 2005). Surprisingly, there were no sex-related differences in the thalamus or the cortex gray matter volume in RI mice. In humans, males tend to have larger cortex gray matter volumes (Sullivan et al., 2004); thus, our finding of no sex-related difference in RI mice may be related to species differences or insufficient sensitivity of our methods to detect subtle volume differences.

As an exploratory effort, we examined the relationship between archival behavioral data and the volume measures. The selected behaviors included spatial memory (Morris water maze performance), fear conditioning, exploration during the light-dark paradigm, exploratory locomotor activity and sensorimotor gating, because of plausible relationships between these behaviors, thalamic-cortical function and neuro-psychiatric disorders. Recent work has used RI strains to identify QTLs associated with a variety of behavioral phenotypes related to schizophrenia, including prepulse inhibition of the acoustic startle response (Milhaud et al., 2002). Using archival data available on the GeneNetwork, we found a correlation between thalamus volume and spatial memory, as measured by the Morris water maze test. We also observed a correlation between genotype at marker D16Mit100 and spatial memory using a small number of strains $(n=11)$. This finding is particularly interesting given the results of human studies reporting deficits in spatial memory in subjects with schizophrenia (Waldo et al., 2005) and the velocardiofacial syndrome (22q11 deletion syndrome) (Bearden et al., 2001).

Although we did not find an overall genetic correlation between prepulse inhibition of the acoustic startle response and thalamus or cortex gray matter volumes, we did find correlations between prepulse inhibition of the acoustic startle response and genotype (Fgd4, Tnfrsf17) at the thalamus and cortex gray matter volume QTL within peak marker D16Mit100 and genotype (2900060K15Rik) at the cortex gray matter volume QTL within peak marker D2Mit42 (see Tables 2 and 3). Considering this discrepancy, it is possible the genes related to both behavioral measures and structural volumes are present within these QTLs, but exert only a weak effect on the behavioral phenotypes. Alternatively, it is possible that relationships between these anatomical and behavioral phenotypes are mediated by other susceptibility loci not segregating in this cross.

There are numerous structural and functional connections between thalamus and cerebral cortex (Krettek and Price, 1977; Groenewegen, 1988; Scannell et al., 1999; Shipp, 2003; Matsuzaki et al., 2004). Evidence from studies of schizophrenia subjects, as well as relevant animal models, suggests abnormalities in the circuitry between the thalamus (especially the mediodorsal nucleus) and the prefrontal cortex (Egan et al., 2001; Volk and Lewis, 2003). In fact, the cognitive deficits and symptoms of schizophrenia have been hypothesized to be caused, at least in part, by a failure of the coordinated function of the thalamus and cortex. Although it remains unclear whether such abnormalities develop because of genetic or environmental influences, our results support the plausibility of genetic influences. In particular, our 
results suggest that shared genetic influences modulate thalamus and cortex gray matter volumes, possibly in conjunction with an influence on spatial memory function in the mammalian brain. Because of the role of the thalamus as a relay and filter station in the brain, modulating input from many cortical areas as well as from the reticular activating and limbic system, it is not surprising that the development of the thalamus shares some common genetic influences with the cortex (Jones and Friedman, 1982; Giguere and Goldman-Rakic, 1988).

A QTL that was associated with cortex gray matter volume (within the peak marker D19Mit40) was also correlated with the expression of AKT1. Recently, altered AKT1-GSK3 $\beta$ signaling has been implicated in the pathogenesis of schizophrenia (Emamian et al., 2004). Moreover, polymorphisms of this gene may confer increased risk for the development of schizophrenia (Ikeda et al., 2004; Schwab et al., 2005; Bajestan et al., 2006). Although the mechanisms linking AKT1 expression to schizophrenia or brain development are unknown, it is intriguing to consider its role in neuroprotection (Dhandapani et al., 2005; Zhang et al., 2005).

Our major finding was that a single QTL on chromosome 16 was correlated with both thalamus and cortex gray matter volumes. Interestingly, this QTL is in a region syntenic to 22q11, a susceptibility locus for schizophrenia. Furthermore, velocardiofacial syndrome linked to $22 \mathrm{q} 22$ is also associated with structural abnormalities in the thalamus and the cortex (Campbell et al., 2006), as well as psychotic symptoms (Murphy et al., 1999). An interesting candidate gene in the vicinity of D16Mit100 is the reticulon 4 receptor gene ( $\operatorname{tn} 4 R)$, located $6.1 \mathrm{Mb}$ from the peak. In humans, this gene is located at 22q11 (Sinibaldi et al., 2004), near the recent schizophrenia candidate genes encoding $\mathrm{PRODH}$ and COMT (Fan et al., 2003; Williams et al., 2003; Lee et al., 2005). $R \operatorname{tn} 4 R$ is an attractive candidate gene, as it has been observed to play a role in the inhibition of neurite elongation (Oertle et al., 2003). Moreover, an association has been shown between $R \operatorname{tn} 4 R$ gene mutations and the risk for developing schizophrenia (Sinibaldi et al., 2004). These findings support the hypothesis that genetically influenced abnormalities in the coordinated development of the thalamus and cortex may lead to psychotic symptoms in several neuropsychiatric disorders.

We cannot rule out the possibility that the QTLs identified could have had some influence on brain tissue density or the degree of tissue shrinkage during fixation instead of brain volume per se, as we measured the volumes after histological processing. In future studies, fresh brain volumes could be measured using Archimedes' rule, and volumes estimated using our present methods and this alternative method could be compared (Dorph-Petersen et al., 2005). Although the total mouse brain volume could have been measured at harvesting, it would have been difficult to obtain accurate fresh subcortical regional volumes because of random error in the hand dissection of small structures. Future developments in mouse brain imaging could redress the current problems caused by tissue shrinkage in preparing brains for measurement.

Given the small number of strains (34 BXD strains) that were used for this study, we did not think we had enough degree of freedom for mapping of epistasis. As more BXD strains are generated and added to the Mouse Brain Library (up to 58 strains), the number of strains will be high enough to allow us to identify epistasis. Meanwhile, we can replicate our earlier mapping results using the new strains.

To summarize we have identified separate and shared genetic influences on thalamus and cortex gray matter volumes. Our results suggest that a gene(s) near marker D16Mit100 may have pleiotropic effects on the structure and function of the thalamus and cerebral cortex. Another potential candidate gene for the cortex volume alone was found on chromosome 19. The QTL with a shared influence on thalamus and cortex gray matter volumes is in a region syntenic to susceptibility locus for schizophrenia. These results suggest that polymorphisms of genes within this region should be investigated for their potential effects on the risk of developing psychiatric disorders associated with disturbances of the development and function of the thalamus and cerebral cortex.

\section{Acknowledgements}

This work was supported by PHS Grants MH071616 (JGC and JMC) NIAAA (U01AA13499 and U24AA13513), NIDA P20-DA 21131, BIRN NCRR U01NR 105417 and NCI MMHCC U01CA105417 (RWW) and U01AA014425 (LL).

\section{References}

Bajestan SN, Sabouri AH, Nakamura M, Takashima H, Keikhaee MR, Behdani F et al. (2006). Association of AKT1 haplotype with the risk of schizophrenia in Iranian population. Am I Med Genet B Neuropsychiatr Genet 141: 383-386.

Bearden CE, Woodin MF, Wang PP, Moss E, McDonald-McGinn D, Zackai E et al. (2001). The neurocognitive phenotype of the 22q22.2 deletion syndrome: selective deficit in visual-spatial memory. J Clin Exp Neuropsychol 23: 447-464.

Carvell GE, Simons DJ (1987). Thalamic and corticocortical connections of the second somatic sensory area of the mouse. J Comp Neurol 265: 409-427.

Campbell LE, Daly E, Toal F, Stevens A, Azuma R, Catani M et al. (2006). Brain and behaviour in children with $22 \mathrm{q} 11.2$ deletion syndrome: a volumetric and voxel-based morphometry MRI study. Brain 129: 218-228.

Chesler EJ, Lu L, Shou S, Qu Y, Gu J, Wang J et al. (2005). Complex trait analysis of gene expression uncovers polygenic and pleiotropic networks that modulate nervous system function. Nat Genet 37: 233-242.

Chesler EJ, Lu L, Wang J, Williams RW, Manly KF (2004). WebQTL: rapid exploratory analysis of gene expression and genetic networks for brain and behavior. Nat Neurosci 7: $485-486$.

Cheverud JM, Ehrich TH, Hrbek T, Kenney JP, Pletscher LS, Semenkovich CF (2004). Quantitative trait loci for obesityand diabetes-related traits and their dietary responses to high-fat feeding in LGXSM recombinant inbred mouse strains. Diabetes 53: 3328-3336.

Dhandapani KM, Wade FM, Wakade C, Mahesh VB, Brann DW (2005). Neuroprotection by stem cell factor in rat cortical neurons involves AKT and NFkappaB. J Neurochem 95: 9-19.

Doerge RW (2002). Mapping and analysis of quantitative trait loci in experimental populations. Nat Rev Genet 3: 43-52.

Dorph-Petersen KA, Pierri JN, Perel JM, Sun Z, Sampson AR, Lewis DA (2005). The influence of chronic exposure to antipsychotic medications on brain size before and after tissue fixation: a comparison of haloperidol and 
olanzapine in macaque monkeys. Neuropsychopharmacology 30: 1649-1661.

Egan MF, Goldberg TE, Kolachana BS, Callicott JH, Mazzanti CM, Straub RE et al. (2001). Effect of COMT Val108/158 Met genotype on frontal lobe function and risk for schizophrenia. Proc Natl Acad Sci USA 98: 6917-6922.

Emamian ES, Hall D, Birnbaum MJ, Karayiorgou M, Gogos JA (2004). Convergent evidence for impaired AKT1-GSK3beta signaling in schizophrenia. Nat Genet 36: 131-137.

Falconer DS, Mackay T (1997). Introduction to Quantitative Genetics. John Wiley \& Sons: NewYork.

Fan JB, Ma J, Zhang CS, Tang JX, Gu NF, Feng GY et al. (2003). A family-based association study of T1945C polymorphism in the praline dehydrogenase gene and schizophrenia in the Chinese population. Neurosci Lett Mar 6 338: 252-254.

Giguere M, Goldman-Rakic PS (1988). Mediodorsal nucleus: areal, laminar, and tangential distribution of afferents and efferents in the frontal lobe of rhesus monkeys. J Comp Neurol 277: 195-213.

Groenewegen HJ (1988). Organization of the afferent connections of the mediodorsal thalamic nucleus in the rat, related to the mediodorsal-prefrontal topography. Neuroscience 24: $379-431$.

Ikeda M, Iwata N, Suzuki T, Kitajima T, Yamanouchi Y, Kinoshita Y et al. (2004). Association of AKT1 with schizophrenia confirmed in a Japanese populations. Biol Psychiatry 24: 698-700.

Ilinsky IA, Kultas-Ilinsky K (1987). Sagittal cytoarchitectonic maps of the Macaca mulatta thalamus with a revised nomenclature of the motor-related nuclei validated by observations on their connectivity. J Comp Neurol 262: 331-364.

Lee SG, Joo Y, Kim B, Chung S, Kim HL, Lee I et al. (2005). Association of Ala72Ser polymorphism with COMT enzyme activity and the risk of schizophrenia in Koreans. Hum Genet 116: 319-328.

James AC, James S, Smith DM, Javaloyes A (2004). Cerebellar, prefrontal cortex, and thalamic volumes over two time points in adolescent-onset schizophrenia. Am J Psychiatry 161: 1023-1029.

Johnson MJ, Alloway KD (1994). Sensory modulation of synchronous thalamocortical interactions in the somatosensory system of cat. Exp Brain Res 102: 181-197.

Jones EG (1997). Cortical development and thalamic pathology. Schizophr Bull 23: 483-501.

Jones EG, Friedman DP (1982). Projection pattern of functional components of thalamic ventrobasal complex on monkey somatosensory cortex. I Neurophysiol 48: 521-544.

Korstanje R, Paigen B (2002). From QTL to gene: the harvest begins. Nat Genet 31: 235-236.

Krettek JE, Price JL (1977). The cortical projections of the mediodorsal nucleus and adjacent thalamic nuclei in the rat. I Comp Neur 171: 157-192.

Kievit J, Kuypers HGJM (1977). Organization of thalamocortical connexions to the frontal lobe in the rhesus monkey. Exp Brain Res 29: 299-322.

Matsuzaki R, Kyuhou S, Matsuura-Nakao K, Gemba H (2004). Thalamo-cortical projections to the posterior parietal cortex in the monkey. Neurosci Lett 355: 113-116.

Mendrek A, Laurens KR, Kiehl KA, Ngan ET, Stip E, Liddle PF (2004). Changes in distributed neural circuitry function in patients with first-episode schizophrenia. Br J Psychiatry 185: 205-214.

Milhaud JM, Halley H, Lassalle JM (2002). Two QTLs located on chromosomes 1 and 5 modulate different aspects of the performance of mice of the $\mathrm{B} \times \mathrm{D}$ Ty RI strain series in the Morris navigation task. Behav Genet 32: 69-78.

Mizuno M, Yamada K, Takei N, Tran MH, He J, Kamei H et al. (2003). Phosphatidylinositol 3-kinase: a molecule mediating BDNF-dependent spatial memory formation. Mol Psychiatry 8: $217-224$.
Murphy KC, Jones LA, Owen MJ (1999). High rates of schizophrenia in adults with velo-cardio-facial syndrome. Arch Gen Psychiatry 56: 940-945.

Oertle T, van der Haar ME, Bandtlow CE, Robeva A, Burfeind P, Buss A et al (2003). Nogo-A inhibits neurite outgrowth and cell spreading with three discrete regions. J Neurosci $\mathbf{2 3}$ 5393-5406.

Paxinos G, Franklin KBJ (2001). The Mouse Brain in Stereotaxic Coordinates. Academic Press: San Diego.

Peirce JL, Chesler EJ, Williams RW, Lu L (2003). Genetic architecture of the mouse hippocampus: identification of gene loci with selective regional effects. Genes Brain Behav 2: 238-252.

Scannell JW, Burns GA, Hilgetag CC, O'Neil MA, Young MP (1999). The connectional organization of the cortico-thalamic system of the cat. Cereb Cortex 9: 277-299.

Schwab SG, Hoefgen B, Hanses C, Hassenbach MB, Albus M, Lerer B et al. (2005). Further evidence for association of variants in the AKT1 gene with schizophrenia in a sample of European sib-pair families. Biol Psychiatry 58: 446-450.

Shipp S (2003). The functional logic of cortico-pulvinar connections. Philos Trans R Soc London B Biol Sci 358: 1605-1624.

Sinibaldi L, De Luca A, Bellacchio E, Conti E, Pasini A, Paloscia C et al. (2004). Mutations of the Nogo-66 receptor (RTN4R) gene in schizophrenia. Hum Mutat 24: 534-535.

Sullivan EV, Rosenbloom M, Serventi KL, Pfefferbaum A (2004). Effects of age and sex on volumes of the thalamus, pons, and cortex. Neurobiol Aging 25: 185-192.

Thompson PM, Cannon TD, Narr KL, van Erp T, Poutanen VP, Huttunen $M$ et al. (2001a). Genetic influences on brain structure. Nat Neurosci 4: 1253-1258.

Thompson PM, Vidal C, Giedd JN, Gochman P, Blumenthal J, Nicolson $\mathrm{R}$ et al. (2001b). Mapping adolescent brain change reveals dynamic wave of accelerated gray matter loss in very early-onset schizophrenia. Proc Natl Acad Sci USA 98 11650-11655.

Volk DW, Lewis DA (2003). Effects of a mediodorsal thalamus lesion on prefrontal inhibitory circuitry: implications for schizophrenia. Biol Psychiatry 53: 385-389.

Waldo MC, Woodward LA, Cawthra EM, Adler LE (2005). The virtual water maze, P50 sensory gating, and cognitive functioning. Schizophr Bull 31: 346.

Walhovd KB, Fjell AM, Reinvang I, Lundervold A, Dale AM, Eilertsen DE et al. (2005). Effects of age on volumes of cortex, white matter and subcortical structures. Neurobiol Aging 26: 1261-1270.

Wang J, Williams RW, Manly KF (2003a). WebQTL: web-based complex trait analysis. Neuroinformatics 1: 299-308.

Wang Q, Liu L, Pei L, Ju W, Ahmadian G, Lu J et al. (2003b). Control of synaptic strength, a novel function of Akt. Neuron 38: 915-928.

Wehner JM, Sleight S, Upchurch M (1990). Hippocampal protein kinase $\mathrm{C}$ activity is reduced in poor spatial learners. Brain Res 523: 181-187.

Williams HJ, Williams N, Spurlock G, Norton N, Zammmit S, Kirov G et al. (2003). Detailed analysis of PRODH and PsPRODH reveals no association with schizophrenia. Am J Med Genet B Neuropsychiatr Genet 120: 42-46.

Williams RW (2000). Mapping genes that modulate mouse brain development: a quantitative genetic approach. Results Probl Cell Differ 30: 21-49.

Williams RW, Gu J, Qi S, Lu L (2001). The genetic structure of recombinant inbred mice: high resolution consensus maps for complex trait analysis. Genome Biol 2: 0046.1-0046.18.

Winterer G, Goldman D (2003). Genetics of human prefrontal function. Brain Res Rev 43: 134-163.

Zhang L, Zhou R, Xiang G (2005). Stepholidine protects against $\mathrm{H} 2 \mathrm{O} 2$ neurotoxicity in rat cortical neurons by activation of Akt. Neurosci Lett 383: 328-332.

Zygourakis CC, Rosen GD (2003). Quantitative trait loci modulate ventricular size in the mouse brain. J Comp Neurol 461: 362-369. 